vol. $25-n^{\circ} 1 \mid 2009$

Nouvelles migrations chinoises en Afrique et en Amérique latine

\title{
Étudier les liens entre les migrations intérieures et internationales en suivant les trajectoires migratoires des Boliviens au Brésil
}

Studying the Links between internal and international Migrations by following

the Trajectories of Bolivian Migrants

Estudiar los vínculos entre las migraciones internas e internacionales siguiendo las trayectorias migratorias de los bolivianos en Brasil

Sylvain Souchaud et Rosana Baeninger

\section{(2) OpenEdition}

Journals

Édition électronique

URL : https://journals.openedition.org/remi/4892

DOI : $10.4000 /$ remi.4892

ISSN : $1777-5418$

Éditeur

Université de Poitiers

Édition imprimée

Date de publication : 1 juin 2009

Pagination : 195-213

ISBN : 978-2-911627-51-4

ISSN : 0765-0752

Référence électronique

Sylvain Souchaud et Rosana Baeninger, «Étudier les liens entre les migrations intérieures et internationales en suivant les trajectoires migratoires des Boliviens au Brésil », Revue européenne des migrations internationales [En ligne], vol. 25 - $\mathrm{n}^{\circ} 1$ | 2009, mis en ligne le 01 juin 2012, consulté le 23 novembre 2021. URL : http://journals.openedition.org/remi/4892 ; DOI : https://doi.org/10.4000/remi. 4892 


\section{Étudier les liens entre les migrations intérieures et internationales en suivant les trajectoires migratoires des Boliviens au Brésil}

\section{Sylvain SOUCHAUD* et Rosana BAENINGER**}

Les changements territoriaux en cours en Amérique du sud seraient de nature à intensifier et diversifier les flux migratoires intra régionaux. En retour, ils établiraient de nouvelles complémentarités spatiales. Nous souhaitons dans cet article explorer cette hypothèse à partir de l'étude des ramifications spatiales de la migration bolivienne au Brésil.

Historiquement, l'immigration bolivienne au Brésil est perçue comme un phénomène de faible intensité situé presque exclusivement aux frontières internationales, à bonne distance des grands centres urbains de la région Sudeste du pays : São Paulo, Rio de Janeiro et Belo Horizonte. Or, récemment semble-t-il, l'immigration bolivienne s'est transformée pour devenir un phénomène majoritairement urbain, métropolitain, tout en conservant un fort ancrage frontalier.

La multiplication et la diversification des lieux de migration nous conduisent à formuler des hypothèses sur l'organisation de ce champ migratoire et d'éventuelles interactions spatiales qui lui confèrent une certaine «efficacité ». Car la population bolivienne immigrée au Brésil, dont les contingents augmentent, présente un profil complexe, en raison de la diversité de ses origines géographiques (des plateaux andins

* Chargé de recherche en géographie, Institut de Recherche pour le Développement (IRD), UMR 151 LPED - France et Centre d'études de la population (NEPO) - Université d'État de Campinas (UNICAMP), São Paulo, Brésil ; sylvain.souchaud@ird.fr

** Professeur, Département de démographie, Institut de philosophie et sciences humaines (IFCH), Centre d'études de la population (NEPO) - Université d'État de Campinas (UNICAMP), São Paulo, Brésil ; baeninge@nepo.unicamp.br 
aux plaines orientales en passant par les vallées inter andines), des lieux de son ancrage au Brésil (de Guajará Mirim, en Amazonie orientale, à São Paulo, à proximité du littoral atlantique) et de la spécialisation des migrants dans certaines activités : la confection textile et le commerce ambulant principalement.

Nous situerons l'étude dans la ville de Corumbá, dans l'État du Mato Grosso du Sud, à la frontière bolivienne. Ville de près de 100000 habitants, Corumbá est non seulement un lieu de concentration de l'immigration bolivienne au Brésil, mais aussi un point d'articulation des circulations à l'intérieur du sous-continent, entre les deux façades atlantiques.

Quelques questions nous orienteront : quel est le poids de Corumbá dans la migration bolivienne, frontalière et non frontalière, au Brésil ? Comment la migration transfrontalière résiste-t-elle dans ses formes traditionnelles à l'évolution des contextes territoriaux ? Corumbá et, plus généralement, l'espace frontalier, sert-il d'espace relais capable de soutenir la formation de courants migratoires boliviens à l'intérieur du Brésil et d'alimenter la migration bolivienne croissante vers São Paulo ?

Nous nous appuierons sur les résultats d'une enquête auprès de 215 ménages d'immigrants boliviens, réalisée en novembre 2006 dans la ville de Corumbá ${ }^{2}$. Les données recueillies nous permettent de considérer les étapes migratoires de différentes populations boliviennes migrantes, aussi bien en Bolivie qu'au Brésil. Dans cet article, nous nous centrons sur les populations installées à Corumbá et nées en Bolivie. Nous accompagnons ainsi le cheminement migratoire, intérieur et international, qui a conduit les migrants jusqu'à leur résidence à Corumbá à la date de référence. Pour compléter notre panorama de l'espace migratoire bolivien, à partir du point d'observation qu'est Corumbá, nous examinons les informations rassemblées sur les membres de la famille (parents, enfants et frères) qui, au moment de l'enquête, n'habitaient pas au domicile du ménage enquêté. En reprenant l'idée que le recours à des solidarités familiales (Boutillier et al., 1977 ; Guilmoto et Sandron, 1999) structure les trajectoires migratoires, nous retenons l'examen des lieux de naissance et de résidence des populations non résidentes afin d'établir des hypothèses sur l'organisation du champ migratoire bolivien au Brésil.

1 L'étude de l'immigration bolivienne à Corumbá est l'un des volets d'un projet de recherche sur les migrations régionales, associant le CNPq (Brésil) et l'IRD (France) et conduit entre 2005 et 2009. La documentation de l'enquête à Corumbá est consultable, en français et en portugais, à l'adresse : http://www.nepo.unicamp.br/fotos/encuestacorumbaout2006.pdf. L'enquête explore un échantillon non représentatif de 215 ménages dont l'un des chefs au moins est né en Bolivie. Au total la population des ménages enquêtés rassemble 958 individus (ceux représentés sur la carte 3), dont 364 sont nés en Bolivie (représentés sur la carte 4). Nous avons également rassemblé des informations sur les enfants des ménages enquêtés ayant quitté le domicile parental (363 individus représentés sur la carte 5). D'autres groupes de parents non résidents sont également étudiés (parents et frères). 


\section{ESPACES FRONTALIERS ET TRAJECTOIRES MIGRATOIRES}

Le rôle des aires frontalières dans l'actuelle géodynamique des migrations internationales sud-américaines évolue, non seulement en raison de l'intensification des échanges migratoires entre pays voisins, mais également grâce à l'incorporation progressive des zones de frontière reculées.

Historiquement, une part importante de la migration internationale entre pays voisins de la région se déploie à la façon d'une migration de voisinage : évènement essentiellement local, la migration internationale restreint géographiquement ses implantations aux espaces frontaliers et est alimentée par des courants originaires d'espaces le plus souvent proches et contigus à cette même frontière.

Les discontinuités spatiales propres au sous-continent expliquent en partie le confinement géographique des échanges migratoires. En effet, à mesure que l'on s'éloigne des centres de pouvoirs situés sur les façades littorales, atlantiques et pacifiques (Volvey et al., 2006) et que l'on s'approche des espaces frontaliers intérieurs, la dispersion et l'enclavement des lieux de peuplement s'accentuent et ont pour effet de limiter la circulation des populations à des aires restreintes. Les migrations internationales n'échappent pas aux contraintes de cet ordre territorial. Demeurant globalement à l'écart des dynamiques continentales, elles n'établissent (ou n'établissaient), par conséquent, pas ou peu de circulations à l'échelle régionale, continentale.

Cependant, malgré l'isolement des frontières et en dépit du caractère local de la migration internationale entre pays voisins, il existe, parmi les habitants frontaliers, une minorité qui sait profiter de l'espace frontalier et élargir ponctuellement ses réseaux jusqu'à des lieux centraux et distants, afin de développer des activités commerciales légales ou illégales (commerce et élevage de bétail, par exemple) (Pébayle, 1978). Ces cas, bien qu'isolés et atypiques, méritent d'être mentionnés, car ils rappellent que les circulations individuelles à l'échelle du sous-continent existent de longue date et soulignent que des pratiques spatiales en apparence opposées, associant courte et longue distance, se structurent aux frontières.

Aujourd'hui encore, d'importantes discontinuités parcourent l'immensité de la frontière du Brésil avec la Bolivie où se côtoient de vastes zones peu occupées et enclavées et des lieux, peu nombreux, hautement intégrés (Carte 1). À la faveur d'une amélioration des moyens de communication, mais plus sûrement grâce à la redistribution progressive des populations dans l'espace national, et principalement vers les espaces intérieurs, certains lieux frontaliers sont au cœur de dispositifs d'échanges continentaux.

Le municipe de Corumbá, territoire de $64961 \mathrm{~km}^{2}$ partageant $200 \mathrm{~km} \mathrm{de}$ frontière avec la Bolivie, illustre cette situation contrastée. Sa population compte 95700 habitants en 2000, dont 84900 installés (IBGE, 2002) dans la zone urbaine de la capitale administrative, Corumbá, à peu de kilomètres de la frontière internationale. Avec une population, en 2000, de 10813 habitants, et une densité de population de $0,17 \mathrm{hab} . / \mathrm{km}^{2}$, la zone rurale du municipe de Corumbá est à n'en pas douter une frange 
peu intégrée. Par opposition, la ville de Corumbá fait figure de point nodal dans l'organisation des échanges et la dynamique d'intégration du sous-continent (Oliveira, 1998). La ville est un lieu central pour l'articulation des flux de personnes, de biens et d'informations, elle est essentielle au dispositif territorial que l'on nomme corridor bi océanique. Cet axe d'intégration (il en existe plusieurs de disposition identique) réunit les deux franges littorales du continent, du sud-est brésilien aux ports péruviens et chiliens du Pacifique, en passant par l'axe de concentration du peuplement Bolivien, Santa Cruz - Cochabamba - La Paz. Corumbá puise son importance, non seulement de sa situation à la frontière internationale, mais aussi parce qu'elle est le principal centre urbain sur une trajectoire de plus de $1000 \mathrm{~km}$, entre Campo Grande, capitale de l'état brésilien du Mato Grosso do Sul, et Santa Cruz de la Sierra, capitale du département bolivien du même nom.

\section{Carte 1 : Localisation}

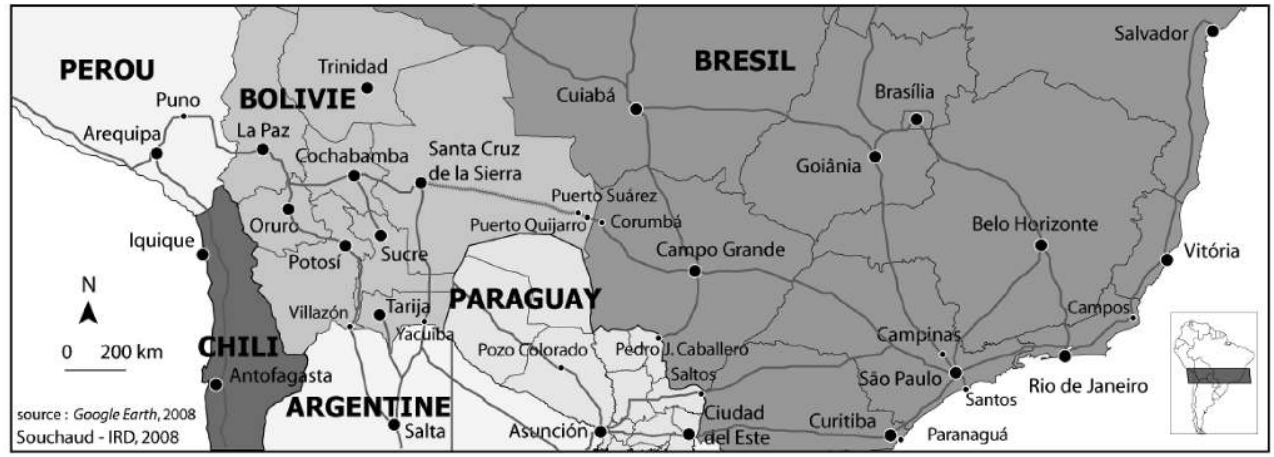

Dans la première moitié du $\mathrm{XX}^{\mathrm{e}}$ siècle, Corumbá n'était pas à proprement parler enclavée, car, depuis plusieurs siècles, elle entretenait (Souchaud et al., 2007) des échanges avec la façade atlantique (avec Rio de Janeiro, principalement, ralliée par les fleuves Paraguay puis Paraná, le bassin de La Plata et le littoral atlantique). C'est au cours des cinquante dernières années que les articulations évoluent grâce à la construction de routes et de voies ferrées dans l'intérieur du sous-continent ; l'intégration territoriale de Corumbá s'effectue désormais selon un axe est-ouest et non plus nord-sud, si bien que les liaisons et les échanges avec la Bolivie s'améliorent et se multiplient.

La circulation le long de ce corridor international ne se limite pas aux marchandises, aux productions agricoles ou minières ; la migration constitue l'un des ressorts de cette dynamique puisque, depuis les années 1950, un vaste mouvement migratoire anime la population bolivienne contribuant à la redistribution de la population vers les départements orientaux. Du côté brésilien, la frontière agricole progresse dans l'État du Mato Grosso do $\mathrm{Sul}^{2}$ alimentant d'intenses mouvements de redistribution et d'installation de populations dans l'intérieur du sous-continent.

2 État dont la création, par division de l'état de Mato Grosso, remonte à 1977. 
L'émigration bolivienne vers le Brésil, dont l'apparition et la croissance s'observent à partir des années 1950 , s'inscrit probablement dans ce vaste mouvement continental de redistribution de la population vers les espaces intérieurs. Ainsi, à cette période, Corumbá voit croître rapidement sa population immigrée, dont les effectifs augmentent à partir de la construction, dans les années 1950, de la ligne de chemin de fer assurant la liaison internationale entre Santa Cruz de la Sierra et le São Pauloº .

\section{LOCALISATIONS ET ITINÉRAIRES DES BOLIVIENS AU BRÉSIL}

En 2000, le recensement de la population enregistrait 20400 immigrants boliviens ${ }^{4}$ sur le territoire brésilien, population en augmentation de $23 \%$ depuis 1991 (ils étaient alors 15 694). C'est apparemment peu, même si on considère que le Brésil n'est pas actuellement un pays d'immigration (autour de 624000 immigrants dans l'ensemble du pays en 2000, selon le recensement $)^{5}$. Mais il est probable qu'une part significative de la population immigrante bolivienne échappe aux recensements brésiliens : population en grande partie pauvre, elle se trouve dans une situation de fragilité qui l'expose davantage à la clandestinité. La plus grande partie de cette population immigrée est installée dans la ville de São Paulo ; selon les estimations de la Pastorale des Migrants, entre 150000 et 200000 Boliviens seraient en situation irrégulière dans le grand São Paulo (Bassegio et Udovic, 2006). Ces chiffres sont probablement surestimés, car ils signifieraient qu'à eux seuls les Boliviens sans papiers de la région métropolitaine de São Paulo seraient 7 à 10 fois plus nombreux que le total des Boliviens recensés au Brésil en 2000. En outre, ils représenteraient de 1,81\% à $2,42 \%$ de la population totale de la Bolivie en 2001 (Instituto Nacional de Estadística, $2003)^{6}$.

On rappellera enfin que la récente régularisation ${ }^{7}$ a permis de sortir de l'illégalité quelques 42000 immigrants boliviens, principalement installés à São Paulo. Cependant tous les immigrants et pour différentes raisons n'ont pu bénéficier de la possibilité de régularisation : peur de sortir de l'anonymat, entraves administratives au

3 Aujourd'hui la liaison ferroviaire est toujours active côté bolivien, la ligne est désaffectée côté brésilien.

4 On se limite ici aux individus nés en Bolivie, résidant et recensés au Brésil. Les immigrants boliviens ne bénéficient pas des facilités d'installation et de circulation accordées aux ressortissants de pays voisins membres du Mercosud. En revanche, la naissance d'un enfant sur le territoire brésilien permet aux parents d'obtenir un titre de séjour permanent. Cette possibilité permet la régularisation de nombreux migrants.

5 Avec plus de 213000 individus nés au Portugal, la population portugaise constitue encore aujourd'hui, en volume, la principale population immigrée au Brésil ; elle représente $31 \%$ de la population totale née à l'étranger.

6 La population bolivienne était de 8274325 habitants en 2001.

7 Procédure engagée dans le cadre de l'exécution d'un accord bilatéral signé en 2005. 
Carte 2 : Population née en Bolivie selon le munícipio de résidence en 2000

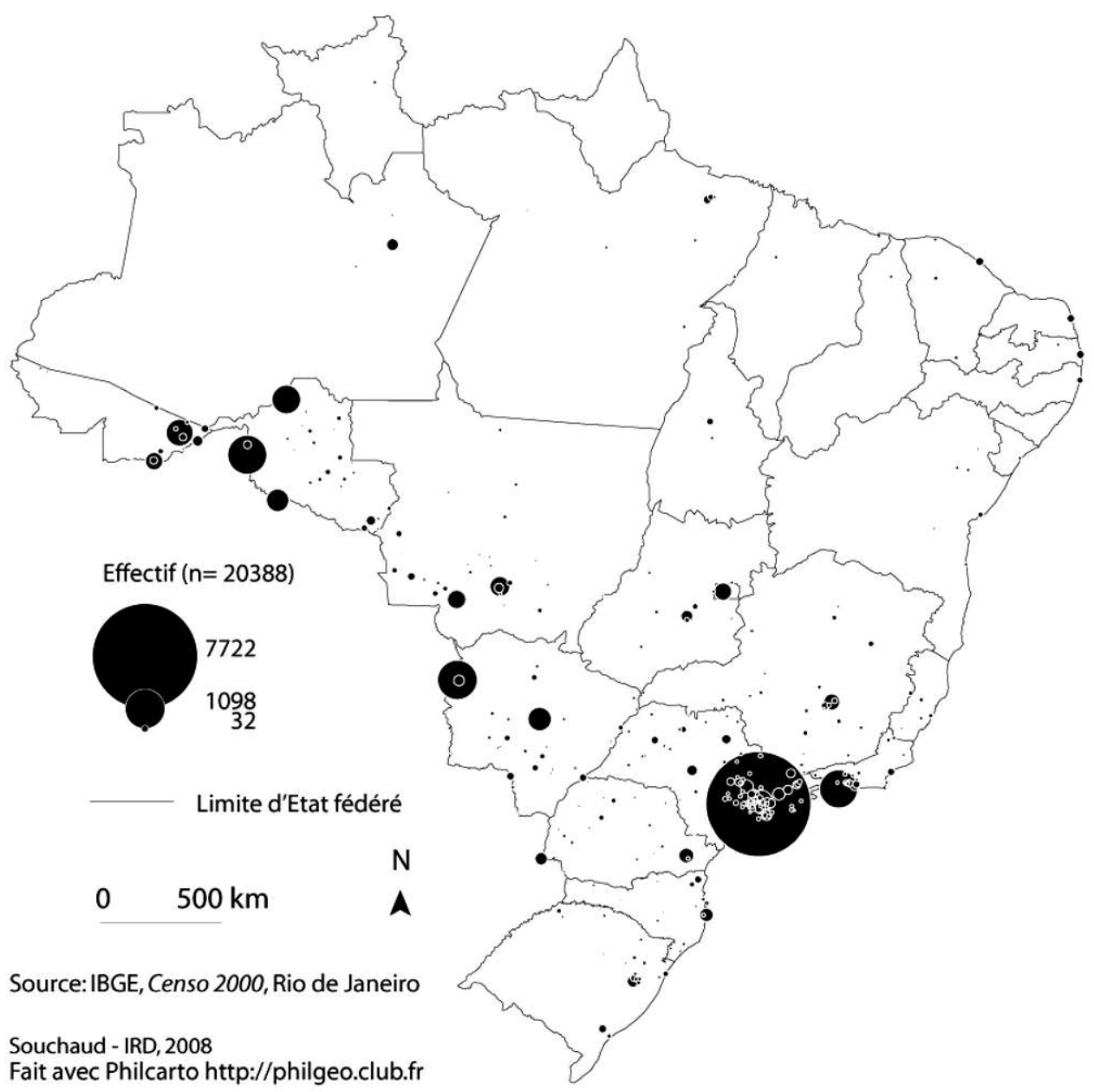

Brésil et en Bolivie, coût de l'opération. Néanmoins si on ajoute au nombre de régularisés le stock de personnes recensées on enregistre près de 100000 individus sur l'ensemble du pays. Reste qu'il semble impossible d'évaluer la présence bolivienne au Brésil. Par contre l'information intéressante que nous livrent les chiffres du recensement concerne les caractères de la répartition spatiale de ce groupe. Car on peut bien admettre qu'il est possible de décrire avec une certaine justesse une population alors même qu'on ne parvient pas à en établir le nombre exact. C'est le cas de la population bolivienne de São Paulo, largement sous-enregistrée (7 722 individus en 2000 selon le recencement), dont les principales caractéristiques enregistrées par le recensement, distribution spatiale, activité, durée de résidence, etc. semblent confirmées par des travaux empiriques (Cymbalista et Xavier, 2007 ; Silva, 1997). La distribution de la 
population bolivienne à l'échelle du Brésil proposée par l'Institut Brésilien de Géographie et Statistiques (IBGE) reflète assez fidèlement les principaux équilibres de cette répartition que nous avons observés ${ }^{8}$.

La carte 2 montre tout d'abord une présence bolivienne dans tous les États du Brésil à l'exception de l'Amapá, à l'extrême nord du pays. Cependant, l'immigration bolivienne est plus significative dans les zones de frontières et dans les régions métropolitaines (principalement à Sao Paulo, et dans une moindre mesure, Rio de Janeiro). Mais cette dispersion sur le territoire et en des lieux si différents, des périphéries orientales aux métropoles du Sudeste, est toutefois relative car on observe, aux frontières comme dans les métropoles, une localisation sélective. Ainsi, la présence à la frontière s'accentue en deux points distants sur la carte : dans le municipe de Guajará-Mirim, au nord, et dans celui de Corumbá, à plus $1200 \mathrm{~km}$ au sud. La distribution de l'immigration bolivienne dans l'espace frontalier brésilien reproduit et même renforce les caractéristiques d'une région marquée par la faible densité d'une population concentrée dans quelques centres urbains dispersés le long de la frontière internationale. Dans le municipe de Corumbá, les Boliviens se regroupent dans le cheflieu du même nom, de la même façon que les Brésiliens se concentrent dans l'espace urbain, délaissant les vastes aires rurales semi désertes.

Par ailleurs, l'attractivité métropolitaine pour la population bolivienne n'est valable que pour Rio de Janeiro, et surtout São Paulo. Et si on ajoute que Guajará Mirim et Corumbá sont les deux «municipes » disposant d'une structure urbaine limitrophe sur l'ensemble de l'espace frontalier boliviano brésilien, on comprend que la répartition de la population bolivienne en territoire brésilien obéit avant tout à une prédilection urbaine, limitée aux principaux centres urbains majeurs, quel que soit le type d'espace, marge frontalière intérieure ou métropole des régions littorales.

À ce stade, on saisit les raisons qui président à l'installation dans chacun des principaux espaces d'immigration, frontière ou métropole, en les considérant séparément, mais on ne dissipe pas l'incompréhension du cadre général : c'est en effet un contraste singulier que l'immigration bolivienne souligne par sa présence, entre Corumbá, en plein cœur du Pantanal, vaste plaine inondable de très faible densité, d'une part, et São Paulo, principale métropole du sous-continent, d'autre part. En 2000, São Paulo rassemble $38 \%$ de la population totale née en Bolivie (Censo IBGE, 2000). Quant à Corumbá, elle ne concentre que $5 \%$ de l'immigration totale ; c'est pourtant le deuxième pôle migratoire bolivien au Brésil.

La distribution spatiale de la migration bolivienne au Brésil s'opère selon deux modalités que résument les formules suivantes : une «migration de voisinage », concernant Corumbá, et une "migration métropolitaine exclusive », concernant São Paulo ${ }^{9}$.

8 Nous nous référons aux lieux où nous avons séjourné et étudié cette population : São Paulo, Corumbá, Ladário, Guajará-Mirim.

9 Pour l'exposé de quelques éléments d'une typologie des espaces migratoires dans la région, dont les deux termes utilisés ici : Souchaud S., Carmo R. Luiz do, Fusco W. «Mobilidade Populacional e Migração no Mercosul : A fronteira do Brasil com Bolívia e Paraguai ». Teoria \& Pesquisa, 2007, XVI (1), pp. 39-60. 
Reste à savoir s'il existe, dans le cadre de la migration bolivienne, une liaison entre ces deux espaces : liaison à la fois improbable, compte tenu du contraste souligné précédemment, et inévitable compte tenu de ce que l'on a exposé plus haut au sujet de l'intégration des espaces intérieurs, de l'activation de liaisons bi océaniques et de l'intensification des échanges et des circulations sur ces axes. À l'échelle locale, rien ne rapproche les villes de Corumbá et São Paulo, mais si l'on examine l'échelon régional de la dynamique spatiale, en rappelant qu'elle se réalise principalement grâce à la mise en réseau des villes, alors il est légitime de poser l'interaction urbaine Corumbá-São Paulo comme un élément structurant le champ migratoire bolivien.

Deux questions se posent. La première, porte sur les origines géographiques du flux migratoire installé à Corumbá, la seconde, sur les extensions spatiales de cette même migration. Les réponses apportées nous permettront de définir, avec plus ou moins de précision, le périmètre du bassin migratoire en amont et en aval de Corumbá. On devrait pouvoir ainsi préciser la place et le rôle de Corumbá et de sa population migrante au sein du vaste champ migratoire bolivien au Brésil.

\section{MIGRATION INTERNATIONALE COMME CONTINUITÉ SPATIALE DE LA MIGRATION INTERNE}

Le recensement brésilien de la population est la première piste à explorer. Elle ne nous mène cependant pas bien loin, pour plusieurs raisons. Le recensement combine deux questionnaires, l'un appliqué à l'ensemble des ménages, l'autre à un échantillon d'environ $10 \%$ de la population totale. Or, les variables migratoires concernent seulement la population de l'échantillon et, si l'on ajoute que certaines populations migrantes, dont les Boliviens, sont nettement sous-enregistrées, on perçoit la fragilité de l'information censitaire quand elle porte sur de petits effectifs. En 2000, selon le recensement (IBGE, 2002), Corumbá comptait 1098 immigrants boliviens. Mais en réalité, ce nombre est obtenu par extrapolation des 107 immigrants (personnes déclarant être nées en Bolivie) réellement interrogés. C'est un échantillon très faible qui limite les possibilités d'analyses fiables.

Nous avons cherché à connaître le lieu de résidence antérieure de ces immigrants. Deux variables sont concernées, l'une précisant le lieu de résidence antérieure dans les dix dernières années, l'autre précisant le lieu de résidence au 31 juillet 1995. Deux précisions s'imposent. La première est liée à la désagrégation des lieux à l'extérieur du pays, limitée au nom de pays même. Il est donc impossible de connaître les départements boliviens d'origine de nos migrants. La deuxième se rapporte également à la désagrégation de l'information des lieux, à l'intérieur du Brésil cette fois : elle change selon les variables ; ainsi, on peut connaître jusqu'au municipe de résidence au 31 juillet 1995, mais seulement l'État fédéral d'installation dans le cas de l'enregistrement du dernier lieu de résidence au cours des dix années écoulées.

Les réponses apportées par les 107 immigrants boliviens interrogés en 2000 dans le cadre du recensement à la question portant sur leur lieu de dernière résidence au cours de la période 1990-2000 donnent les résultats suivants : 72 individus sont non migrants ou résidents depuis 10 ans dans l'État du Mato Grosso do Sul, 28 déclarent une résidence en Bolivie (sans qu'on puisse préciser le lieu), 1 au Paraguay, 3 dans l'État de São Paulo (sans qu'il soit possible d'identifier le municipe) et 2 dans l'État 
voisin du Mato Grosso. Si l'on examine la variable précisant le municipe de résidence en 1995, on apprend que deux migrants résidaient dans l'intérieur de l'État de São Paulo en 1995. Dès lors on peut conclure que le recensement ne nous permet pas d'étudier les trajectoires migratoires des migrants boliviens installés à Corumbá. Nous utiliserons donc les données de l'enquête réalisée en 2006.

Intéressons-nous tout d'abord aux lieux de naissance des individus intégrant les 215 ménages interrogés lors de l'enquête ENCOR. Rappelons qu'ils sont constitués de populations nées en Bolivie et au Brésil. Lors de l'enquête, qui s'est déroulée en octobre 2006, le critère de sélection des ménages était qu'un chef au moins du ménage soit né en Bolivie. Ainsi, la population totale enquêtée était de 968 individus ${ }^{10}$ (215 ménages), dont 364 étaient nés en Bolivie et 598 au Brésil.

Si on se réfère à la carte 3 , on constate tout d'abord que les Brésiliens intégrants des ménages d'immigrant(s) international(aux) sont fortement ancrés localement. Ils sont, dans une très large proportion, nés à Corumbá même : 549 personnes, soit plus de la moitié de la population totale enquêtée. Seuls quelques-uns sont à bonne distance de la frontière, à Campo Grande, la capitale de l'État de Mato Grosso do Sul, dans le Mato Grosso et dans l'intérieur de l'État de São Paulo. D'autres encore, très peu, sont nés dans le Nordeste du pays, à plusieurs milliers de kilomètres.

La très faible dispersion géographique et l'extrême concentration frontalière des lieux de naissance de la population brésilienne des ménages enquêtés contraste avec la variété des origines géographiques de la population bolivienne. Les immigrants boliviens de Corumbá sont originaires de tous les départements boliviens (Tableau 1), à

Tableau 1 : Département ou État de naissance des habitants des ménages enquêtés à Corumbá

\begin{tabular}{|l|r|r|r|}
\hline Département/État & Effectifs & \% valide & \% cumulé \\
\hline Santa Cruz (Sc) & 269 & 27,9 & 27,9 \\
Cochabamba (Co) & 28 & 2,9 & 30,8 \\
La Paz (Pa) & 41 & 4,3 & 35,1 \\
Oruro (Or) & 6 & 0,6 & 35,7 \\
Potosí(Po) & 10 & 1,0 & 36,7 \\
Beni (Be) & 3 & 0,3 & 37,0 \\
Chuquisaca (Ch) & 3 & 0,3 & 37,3 \\
Tarija (Ta) & 2 & 0,2 & 37,6 \\
Rondônia (Ro) & 2 & 0,2 & 37,8 \\
Ceará (Ce) & 2 & 0,2 & 38,0 \\
Paraíba (Pb) & 1 & 0,1 & 38,1 \\
Pernambuco (Pe) & 1 & 0,1 & 38,2 \\
Rio de Janeiro (Rj) & 1 & 0,1 & 38,3 \\
São Paulo (Sp) & 6 & 0,6 & 38,9 \\
Paraná (Pr) & 3 & 0,3 & 39,2 \\
Mato Grosso do Sul (Ms) & 576 & 59,8 & 99,0 \\
Mato Grosso (Mt) & 4 & 0,4 & 99,4 \\
Distrito Federal (Df) & 1 & 0,1 & 99,5 \\
Ne sait pas & 5 & 0,5 & 100,0 \\
\hline Total & $\mathbf{9 6 4}$ & $\mathbf{1 0 0 , 0}$ & \\
\hline
\end{tabular}

Source : Enquête Encor, Corumbá, 2006.

10 Soit 4 de moins que la population totale, 2 personnes étant nées en Argentine, 2 autres au Pérou. 
Carte 3 : Lieux de naissance de la population résidant à Corumbá en octobre 2006

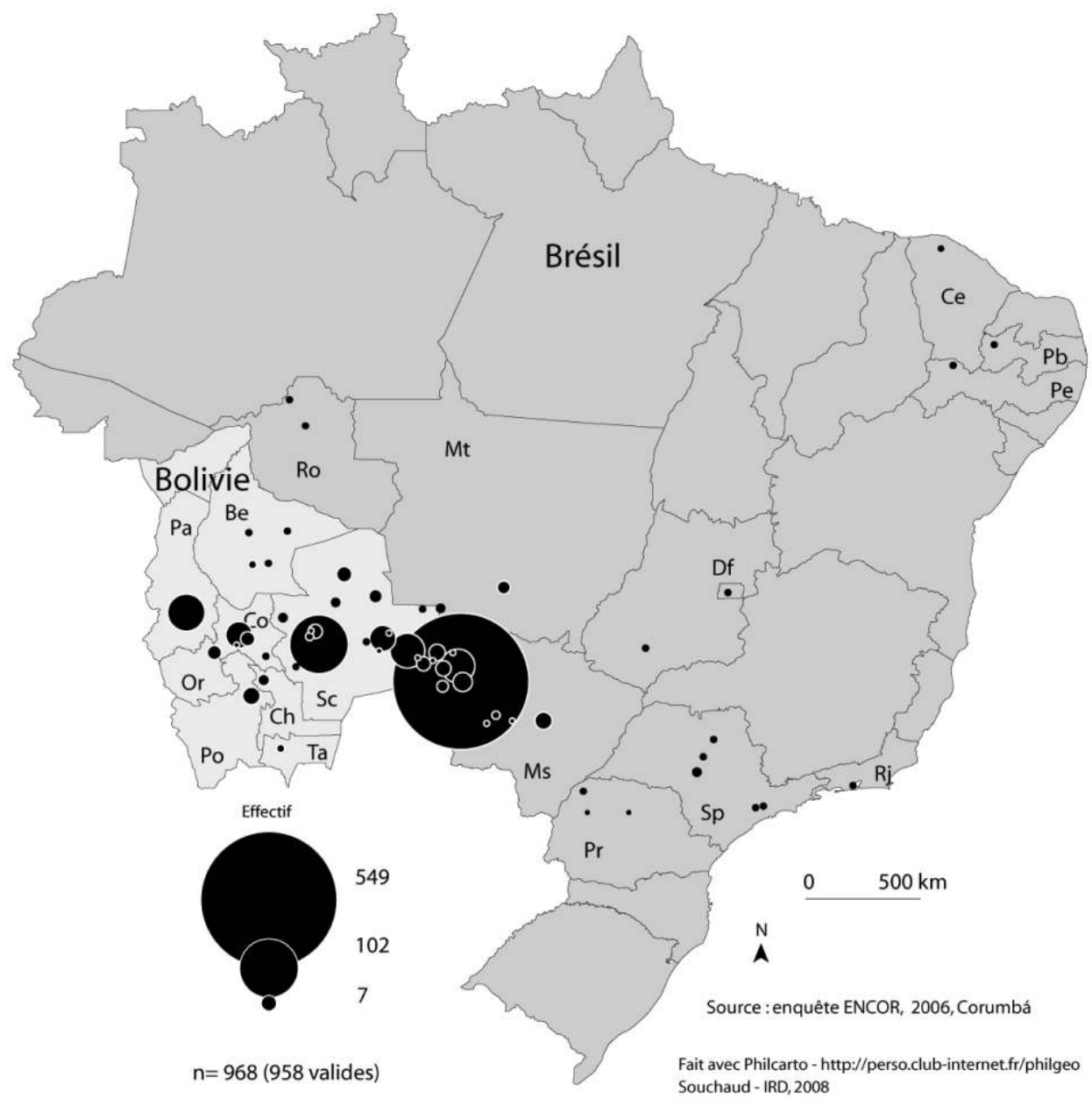

l'exception du Pando, à l'extrême nord du pays. Le département de Santa Cruz est le principal département de naissance. Ces trois départements sont les plus peuplés, respectivement 2,35 millions, 2,03 millions et 1,456 million d'habitants en 2001 (INE, 2003), ce qui représente 70,5\% de la population totale du pays. Mais, la bonne place des départements andins de La Paz et Cochabamba est à signaler.

Ces premiers éléments nous indiquent que la migration bolivienne à Corumbá n'est pas à proprement parlé une migration de proximité. La migration transfrontalière de voisinage n'est que marginale. Des migrants sont nés à Puerto Suárez, Puerto Quijarro, El Carmén, etc. c'est-à-dire dans les petites villes qui jalonnent, depuis la frontière internationale, la voie ferrée qui conduit à Santa Cruz de la Sierra. Ceci 
s'explique en partie pour la simple raison que ces espaces d'origine ont de faibles, voire de très faibles, densités et volumes de population et ne peuvent, par conséquent, alimenter un flux de migration international volumineux. Les provinces boliviennes ont une population peu nombreuse et, de manière générale, l'Est bolivien, jusqu'à Santa Cruz, c'est-à-dire environ $600 \mathrm{~km}$ de frontière, est une zone presque déserte démographiquement.

La proximité n'étant pas l'élément qui organise la dynamique migratoire, on s'aperçoit, à l'examen approfondi des lieux de naissance des migrants que l'élément déterminant est l'origine urbaine, puisque 313 migrants (sur 358 réponses valides) boliviens sont nés dans un centre urbain. On remarque de surcroît que ce sont les grands centres urbains de Bolivie : Santa Cruz de la Sierra, La Paz, Cochabamba, Oruro et Potosí, d'un poids nettement supérieur à celui de la ville de Corumbá, qui alimentent cette migration internationale interurbaine ${ }^{11}$. Dans une étude sur la ville bolivienne de Yacuiba, à la frontière argentine, nous avions observé dans le détail ce même processus (Souchaud et Martin, 2007). Certes, Yacuiba étant une ville bolivienne, nous avions associé le processus migratoire qui nourrit sa croissance à une modalité nouvelle de la redistribution interne de la population bolivienne. Corumbá, est bien une ville brésilienne, mais on peut s'interroger sur le rôle qu'elle joue dans le dispositif socio-spatial bolivien, entre articulations urbaines et redistribution démographique.

Nous avons construit une carte représentant les lieux de résidence qui ont immédiatement précédé l'actuelle migration à Corumbá (Carte 4). L'importance du département de Santa Cruz apparaît sans équivoque. S'il représente 38,1 \% du total des lieux de naissance des immigrants interrogés, $89 \%$ du total des immigrants y font une ultime étape dans le cheminement qui les conduit à Corumbá. Le département de Santa Cruz est à la fois un lieu d'origine et une étape dans le parcours migratoire des Boliviens. On remarque par ailleurs que Santa Cruz de la Sierra, la capitale, en raison de son poids démographique, polarise le flux. Mais d'autres espaces d'accueil accentuent leur poids, de petites villes principalement (comme Roboré), situées à l'est de la capitale départementale et situées sur la trajectoire ferroviaire. On constate à nouveau que l'espace frontalier bolivien, stricto sensu, n'est que l'une des nombreuses pièces du dispositif spatial qui structure le phénomène migratoire frontalier.

L'examen de la distribution spatiale d'un événement (l'avant-dernière étape) de l'historique migratoire (Carte 4) montre que l'arrivée à Corumbá est précédée d'une migration interne en Bolivie. Et quelle que soit l'origine géographique du migrant, cette migration interne passe par le département de Santa Cruz.

Ainsi, la migration internationale à Corumbá s'associe au mouvement de redistribution interne de la population que la Bolivie connaît depuis les années 1950, marqué essentiellement par la migration d'importants groupes de population de la

11 Dit autrement, La Paz et Santa cruz de la Sierra sont les deux principaux centres urbains du pays et les plus importants foyers d'émigrations vers Corumbá. 
région andine vers la région basse (Blanchard, 2005 ; D’Andrea, 2004 ; Martin, 2005 ; Perrier-Bruslé, 2005). Le département de Santa Cruz fut, sans aucun doute, celui qui connut la plus grande croissance : sa participation relative au total de la population bolivienne augmentant de $9 \%$ en 1950 à 24,5\% en 2000 (Souchaud, 2008). En dépit de cette progression importante, le département conserve des disparités dans la distribution de sa population, entre Santa Cruz de la Sierra, la capitale, qui concentre $55 \%$ de la population départementale totale en 2000 (2 029471 habitants) et les municipes de l'intérieur. Ainsi, le reste du département est une vaste zone presque déserte, d'où seuls deux espaces de modeste concentration urbaine se distinguent : à la frontière (Porto Suarez, 11600 habitants en 2001 et Puerto Quijarro, 9000 habitants en 2001) et le long de la voie ferrée (Roboré, 9900 habitants en 2001).

\section{Carte 4 : Dernier lieu de résidence de la population née en Bolivie résidant à Corumbá} en octobre 2006

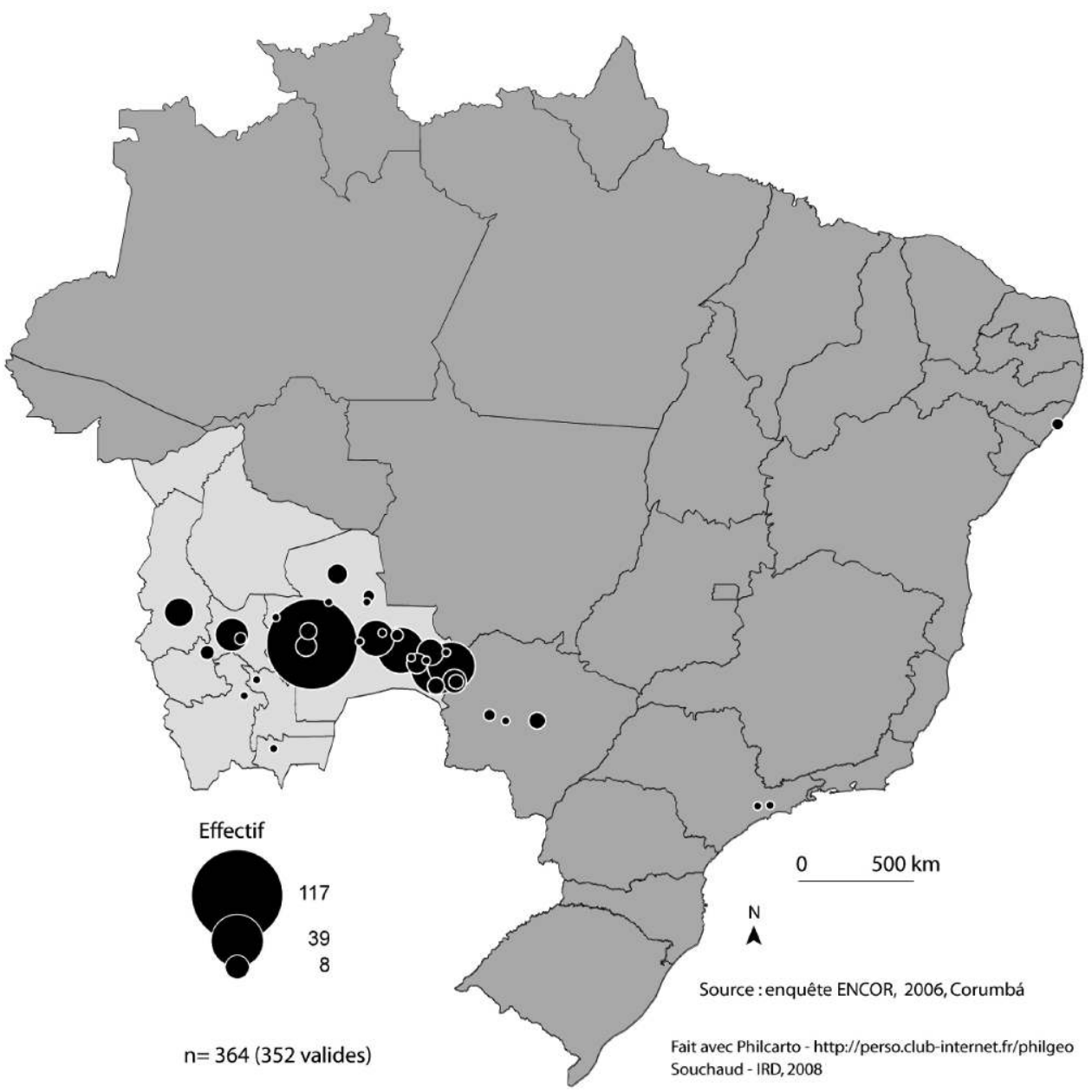




\section{MIGRATION INTERNE DE LA DEUXIÈME GÉNÉRATION : RETOUR EN BOLIVIE ET PREMIẼRE DISPERSION AU BRÉSIL}

S'il est relativement aisé de savoir ce qui se passe avant l'installation dans le lieu d'enquête, il est par contre plus délicat de définir les trajectoires des migrants qui ont quitté ce même lieu. Après avoir traité la migration à Corumbá dans le prolongement de la migration interne, nous tenterons de déterminer si Corumbá alimente une migration interne bolivienne vers d'autres régions du Brésil. Le recensement nous livre peu d'informations à ce sujet, mais il est important de mentionner qu'en 2000, selon celui-ci (micros données de l'échantillon, IBGE, 2002), seulement 37 Boliviens, domiciliés au Brésil en dehors de Corumbá, ont déclaré avoir eu pour lieu de résidence en 1995 le municipe de Corumbá. Voilà qui met en évidence le peu de connexions existantes entre Corumbá et les autres parties du pays et tendrait à indiquer que la population bolivienne de Corumbá n'alimente pas le flux de migration vers la Région Métropolitaine de São Paulo (RMSP), principal courant de la migration bolivienne au Brésil.

L'enquête à Corumbá de 2006 (ENCOR) a permis d'explorer davantage cette voie. D'abord, nous avons cherché à savoir si les enfants de migrants (sortis du domicile parental) avaient eu une expérience migratoire avec leurs parents à l'intérieur du Brésil. Sans entrer dans le détail des parcours migratoires des chefs de famille, il est possible d'envisager la question à partir de l'examen des lieux de naissance des enfants des ménages enquêtés nés au Brésil et ne résidant plus, au moment de l'enquête, au domicile de leurs parents. Or, les enfants qui n'habitent pas avec leurs parents sont nés en majorité à Corumbá : sur un total de 366 enfants non résidents issus des ménages enquêtés, 304 enfants sont nés au Brésil, dont 274 à Corumbá. Si on considère les 16 enfants nés à Ladário (micro municipe enclavé dans le municipe de Corumbá et attenant au périmètre urbain de Corumbá), $95 \%$ des enfants d'immigrants boliviens sortis du foyer parental sont nés dans la zone délimitée par le municipe de Corumbá ${ }^{12}$. Ce résultat tend à montrer que les parents eux-mêmes ont fait peu de migrations à l'intérieur du Brésil, et renforce l'idée de la faible migration interne des Boliviens au Brésil avant l'arrivée à Corumbá. La variable présentée n'épuise pas les possibilités de migrations intercalaires et il n'est pas impossible qu'une migration interne nous échappe, dès lors notamment qu'elle interviendrait à une période précise du cycle de vie : avant la naissance des enfants. Il existe une autre possibilité, celle d'un aller et retour dans le Brésil à partir de Corumbá. Ces situations sont tout à fait envisageables, mais elles ne limitent pas la portée de la donnée rapportée.

12 La proportion est d'ailleurs semblable qu'il s'agisse d'enfants de migrants ayant ou n'ayant pas quitté le domicile parental puisque des 178 enfants corumbaenses (habitants de Corumbá) partageant le domicile parental 148 sont nés au Brésil, dont 142 à Corumbá et 1 à Ladário, soit $97 \%$ du total suivant notre découpage géographique. 
Carte 5 : Lieux de résidence (hors du domicile parental) des enfants de ménages comptant au moins un immigrant, en octobre 2006

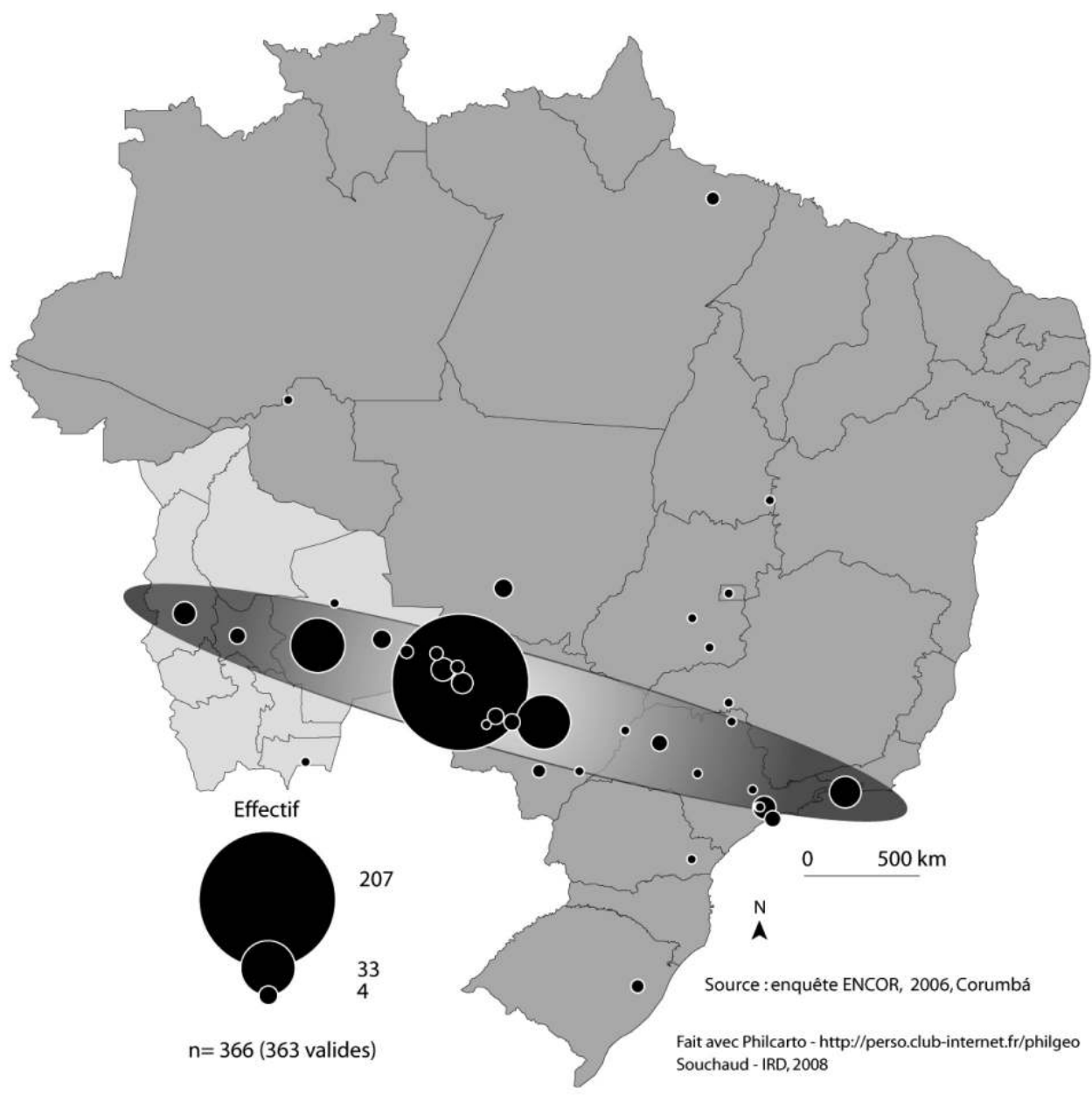

Grâce aux informations recueillies sur les lieux de résidence des enfants qui n'habitent pas habituellement aux domiciles des chefs de famille ${ }^{13}$ de Corumbá, il devient possible de délimiter les espaces migratoires issus de la première vague d'immigration. Cette population ne résume pas elle seule la diversité des parcours migratoires qui s'organisent à partir de Corumbá, mais elle peut annoncer des tendances dans l'organisation spatiale des migrations, peut-être amenées à se renforcer dans le futur.

13 Pour ce travail, on a sélectionné les enfants comme résidents non habituels, même si on pouvait analyser, dans cette catégorie, parents, frères et autres membres de la famille. 
La carte 5, représentant les lieux de résidence actuels des enfants issus des ménages enquêtés à Corumbá, indique qu'une partie significative de cette population habite en Bolivie. Cet aspect est à signaler, car ces enfants de migrants, pour qui le territoire brésilien est librement ouvert dès lors qu'ils sont nés au Brésil (83 \%), sont 60 à habiter en Bolivie, où ils sont immigrants brésiliens ${ }^{14}$.

On retrouve sur la carte la même polarisation des grandes villes situées sur l'axe qui traverse la Bolivie d'est en ouest et, de l'impression générale, ressort le dessin d'un corridor international articulé par les grandes villes de la région (La Paz, Cochabamba, Campo Grande, São Paulo, Rio de Janeiro).

Néanmoins, on observe une mince dispersion de cette population sur le territoire brésilien. La frontière, et plus exactement Corumbá, fixe ces descendants d'immigrants et la ville de São Paulo, principal lieu l'immigration bolivienne, est à peine visible sur la carte. En conséquence, alors qu'il existe de fortes correspondances des schémas de diffusion et de dispersion spatiales d'une génération à l'autre, se confirme l'idée que l'immigration bolivienne à la frontière corumbaense est étrangère à la migration métropolitaine à São Paulo.

\section{CONCLUSION}

L'une de nos interrogations initiales portait sur la réalité de l'immigration frontalière. Pour répondre, il nous faut distinguer deux configurations migratoires à la frontière, selon que l'espace frontalier est un foyer d'immigration et/ou d'émigration.

Le cas de Corumbá fait apparaître nettement que la frontière est attractive pour de nombreux migrants internationaux, dont le choix se fixe sur les principaux centres urbains, Corumbá et Guajará Mirim. On notera que la dynamique migratoire internationale ne coïncide pas avec la dynamique migratoire interne brésilienne. L'attractivité de Corumbá pour les migrants boliviens ne s'applique pas à la population migrante brésilienne, qui s'en détourne. C'est qu'indépendamment de toute attractivité, il faut au migrant un motif.

L'immigration à Corumbá n'est pas une immigration transfrontalière ou, dit autrement, de voisinage. Ainsi, les cantons limitrophes, côté bolivien, ne pourvoient que marginalement à l'immigration frontalière, l'essentiel provenant de la migration en cascade de populations aux origines parfois lointaines. Pour que la situation s'inverse, il faut un état de peuplement consolidé à la frontière. Ce n'est que récemment que l'espace frontalier, côté bolivien, a connu une vague de peuplement conséquente, en mesure d'altérer la logique des échanges migratoires historiques entre l'un et l'autre côté de la frontière internationale.

14 L'enquête de terrain révéla que 300 sont au Brésil, 2 en Espagne, 1 au Pérou, 1 aux ÉtatsUnis, et 1 dans un autre pays non précisé. 
Quant au rôle de Corumbá dans le champ migratoire bolivien au Brésil, plusieurs hypothèses formulées avant de lancer l'enquête à Corumbá ont été abandonnées. Dans un premier temps, nous avancions l'idée que Corumbá serait un espace de transit pour les populations boliviennes immigrant au Brésil. Les facilités administratives qu'accordent les municipes frontaliers à l'installation d'émigrants du pays voisin contrastant avec les difficultés croissantes que les Boliviens doivent surmonter pour atteindre la métropole de São Paulo nous conduisirent à penser que les migrants boliviens pourraient faire étape à Corumbá, pour une durée variable, afin d'y organiser la suite d'un parcours migratoire où São Paulo serait l'étape finale.

Les premiers entretiens réalisés auprès de migrants à Corumbá avant l'enquête avaient déjà ébranlé cette hypothèse et nous avons montré ici qu'il n'y avait presque aucun lien entre Corumbá et São Paulo, que ce soit en amont de l'étape à Corumbá ou à partir de Corumbá, notamment par l'examen de la dispersion spatiale des enfants de migrants ayant quitté le domicile des parents. En réalité, il n'existe aucun effet «communicant » entre la frontière et la métropole, entre Corumbá et São Paulo. Nous cherchions à voir dans la frontière un espace de transit et d'articulation des flux, mais nous ne sommes pas ici sur les marges méridionales de l'Europe et le Pantanal n'est pas le glacis saharien (Bredeloup et Pliez, 2005) où s'organisent d'importantes migrations de transit ${ }^{15}$.

L'une des explications possibles de cette disjonction se trouve peut-être dans la faiblesse des liens entre Corumbá et l'axe de pénétration et d'intégration territoriale brésilien, alimenté par l'expansion de la frontière agricole, des régions sud et sud-est en direction des régions nord et centre ouest. Par certains aspects, l'histoire brésilienne de Corumbá marque un net ralentissement lorsque Rio de Janeiro perd son statut de capitale au profit de Brasília (1960). En quelque sorte, et cela semblera paradoxal, Corumbá se serait trouvée affaiblie par la « marche vers l'Ouest » ${ }^{16}$, mouvement encadrant l'intégration territoriale des marges intérieures. Cependant, nous nous sommes attardés sur des comportements individuels et nos conclusions mériteraient d'être soumises à l'étude des logiques migratoires familiales, dont les mécanismes peuvent différer.

Néanmoins, Corumbá est un centre migratoire international, dont le rayonnement dépasse les frontières occidentales du département voisin de Santa Cruz, pour gagner les capitales andines, La Paz ou Potosí. En marge de la dynamique du mouvement d'intégration dominant du centre ouest brésilien, le municipe de Corumbá cherche à activer les liens avec le pays voisins, ainsi que l'exprime des responsables de l'administration municipale et des dirigeants d'entreprises de Corumbá ${ }^{17}$. Il existe bien une dynamique territoriale extra locale à Corumbá dont la portée est transnationale.

15 En l'occurrence de populations sub-sahariennes vers l'Europe occidentale.

16 Politique nationale d'intégration territoriale et de diffusion du peuplement, basée sur la progression, en direction du nord et de l'ouest, de la frontière agricole.

17 Entretiens réalisés tout au long de l'année 2006. 
Influencé par la théorie «push and pull » qui établit que l'attractivité du Brésil et la « répulsivité » de la Bolivie, l'une et l'autre fondées sur des critères socioéconomiques objectifs, est le moteur principal de la migration, nous avions écarté la Bolivie du champ migratoire, une fois la frontière franchie, pensant que l'avantage comparatif du Brésil l'emporterait envers et contre tout à l'heure du choix du migrant. Pourquoi un migrant bolivien installé au Brésil retournerait-il en Bolivie ? Pourquoi ses enfants, nés au Brésil, Brésilien en vertu du jus soli, regarderaient-ils derrière eux, quand l'horizon est plein de l'immensité brésilienne ? Lors des entretiens, nous avons, ici ou là, identifié quelques familles corumbaenses d'origine bolivienne mentionnant un parent installé à São Paulo. Mais ces cas sont des exceptions, et surtout, ils n'impliquent aucune relation particulière. Tout se passe comme si ces deux trajectoires migratoires étaient strictement dissociées, formant au sein d'une même famille deux projets, deux sphères migratoires distinctes dont les liens restent à construire. Et d'une manière générale, les immigrants boliviens à Corumbá tracent une frontière à l'est de Corumbá et articulent leur espace de vie sur un arrière pays bolivien dont Corumbá serait l'ultime jalon.

\section{Références bibliographiques}

BASSEGIO Luiz et UDOVIC Luciane (2006) « Migrações e Senzalas do século 21 », in Rede Social de Justiça e Direitos Humanos, Direitos Humanos no Brasil 2006. Relatório de Rede Social de Justiça e Direitos Humanos, São Paulo, RSJDH, pp. 137-146.

BLANCHARD Sophie (2005) Être " colla » à Santa Cruz : identités et territoires des migrants andins à Santa Cruz de la Sierra (Bolivie), Université de Paris 1, thèse de doctorat en géographie, $460 \mathrm{p}$.

BREDELOUP Sylvie et PLIEZ Olivier (org.) (2005) Migrations entre les deux rives du Sahara, Revue Autrepart, 36, Paris, Armand Colin, 199 p.

BOUTILLIER Jean-Louis, QUESNEL André et VAUGELADE Jacques (1977) Systèmes socioéconomiques Mossi et migrations, Cahiers ORSTOM. Série Sciences Humaines, 14 (4), Paris, Orstom, pp. 361-381.

CYMBALISTA Renato, XAVIER et ROLNIK Iara (2007) A comunidade boliviana em São Paulo: definindo padrões de territorialidade, Cadernos da metrópole, 17, São Paulo, EDUC, pp. 119-133.

D'ANDREA Nicolas (2004) Effets de frontière, migrations et redistribution du peuplement en Bolivie: les dynamiques territoriales dans le département de Tarija. Université d'Aix en Provence, thèse de doctorat en aménagement de l'espace, urbanisme, $425 \mathrm{p}$.

DOMENACH Hervé, CELTON Dora, ARZE Hugo et HAMELIN Philippe (2007) Movilidad y Procesos Migratorios en el Espacio de Frontera Argentino Boliviana, Córdoba, Universidad Nacional de Códoba, 274 p.

GUILMOTO Christophe Z. et SANDRON Frédéric (1999) Approche institutionnelle de la migration dans les pays en voie de développement, Économie rurale, 252, Paris, pp. 47-54.

INSTITUTO BRASILEIRO DE GEOGRAFIA E ESTADÍSTICA (2002) Censo demográfico 2000, FIBGE, Rio de Janeiro.

INSTITUTO NACIONAL DE ESTADÍSTICA (2003) Bolivia : Características sociodemográficas de la población, La Paz, INE, $319 \mathrm{p}$.

MARTIN Ceydric (2005) Complexe migratoire et distribution spatiale de population dans le Sud bolivien. Enquêtes en milieux rural et urbain dans le département du Tarija, thèse de doctorat de démographie, IRD - Université de Paris V, Paris, 703 p. 
OLIVEIRA Tito Carlos MACHADO DE (1998) Uma fronteira para o pôr-do-sol, Campo Grande, Editora UFMS, 147 p.

PEBAYLE Raymond (1978) «Frontières et espaces frontaliers du Brésil méridional », Cahiers des Amériques latines, 18, pp. 33-44.

PERRIER-BRUSLE Laetitia (2005) La dernière frontière. Loin des Andes, trop près du Brésil. La frontière orientale et la construction du territoire en Bolivie, Université de Paris 1 , thèse de doctorat en géographie, $734 \mathrm{p}$.

SILVA Sidney Antônio da (1997) Costurando Sonhos.Trajetória de um grupo de imigrantes bolivianos em São Paulo, São Paulo, Editora Paulinas, 292 p.

SOUCHAUD Sylvain (2008) «Algumas considerações sobre a migração internacional transfronteiriça a partir do caso da migração boliviana em Corumbá, Mato Grosso do Sul » in A.C. Osório do Nascimento, J.V.H. Pereira et Tito Carlos Machado de Oliveira Éds., América Platina : textos escolhidos. v. 1., Campo Grande, Editora UFMS, 268 p.

SOUCHAUD Sylvain, CARMO Roberto LUIZ DO et FUSCO Wilson (2007) « Mobilidade Populacional e Migração no Mercosul: A fronteira do Brasil com Bolívia e Paraguai », Teoria \& Pesquisa, XVI (1), pp. 39-60.

SOUCHAUD Sylvain et MARTIN Ceydric (2007) « Yacuiba, un islote del Chaco boliviano en la mundialización », in Hervé Domenach, Dora Celton, Hugo Arze et Philippe Hamelin Éds., Movilidad y Procesos Migratorios en el Espacio de Frontera Argentina-Bolivia, Córdoba, Universidad Nacional de Córdoba, p. 55-94.

VOLVEY Anne, AMILHAT-SZARY Anne-Laure, PERRIER-BRUSLE Laetitia, SOUCHAUD Sylvain et SIERRA Philippe L'Amérique latine, Paris, Atlande, 215 p. 


\title{
Étudier les liens entre les migrations intérieures et internationales en suivant les trajectoires migratoires des Boliviens au Brésil \\ Sylvain SOUCHAUD et Rosana BAENINGER
}

Les premières migrations internationales des Boliviens au Brésil, à partir des années 1950 , ont eu la frontière pour principale destination. L'augmentation récente du flux migratoire au Brésil coïncide avec une concentration des migrants boliviens dans la métropole de São Paulo. Les contours d'un nouvel espace migratoire bolivien au Brésil se dessinent où la frontière, en dépit de l'importance croissante de la ville de São Paulo dans l'organisation de l'espace migratoire bolivien, conserve un rôle important et spécifique. À partir des données d'une enquête réalisée fin 2006 dans des ménages immigrés de la ville frontalière de Corumbá, dans l'État du Mato Grosso do Sul, nous étudions dans cet article les changements intervenus dans l'organisation spatiale de ce champ migratoire. Nous souhaitons notamment préciser le rôle actuel de l'espace frontalier, historiquement réputé pour concentrer l'immigration internationale voisine, en tant que pôle migratoire et envisager d'éventuelles articulations avec la métropole de São Paulo et l'espace intérieur bolivien.

\section{Studying the Links between internal and international Migrations by following the Trajectories of Bolivian Migrants}

\author{
Sylvain SOUCHAUD and Rosana BAENINGER
}

The increasing bolivian migration flux in Brazil and the concentration of migrants in the metropolitan area of São Paulo display the shape of a new bolivian migratory space in Brazil. Using information from a survey that took place in the border city of Corumbá at the end of 2006 , we study the changes that occurred in the spatial organization of the migratory space we consider. We wish specifically insist on the current role of the border area, historically consider being a place of migration concentration, as a migration pole and his possible relations with the city of São Paulo.

\section{Estudiar los vínculos entre las migraciones internas e internacionales siguiendo las trayectorias migratorias de los bolivianos en Brasil}

\section{Sylvain SOUCHAUD y Rosana BAENINGER}

La migración internacional de los bolivianos en el Brasil tuvo como primer destino, en los años 1950, la frontera internacional. En la actualidad, el crecimiento reciente del flujo migratorio entre Bolivia y Brasil coincide con la concentración de los inmigrantes bolivianos en la región metropolitana de São Paulo. Se esboza entonces un nuevo espacio migratorio de los migrantes bolivianos en el Brasil donde la frontera, a pesar de la importancia del intenso flujo hacia la ciudad en la organización general del flujo internacional, mantiene un papel importante y específico. A partir de una investigación de campo llevada a cabo a finales del 2006 en hogares de inmigrantes bolivianos de la ciudad fronteriza de Corumbá, en el estado brasileño de Mato Grosso do Sul, es posible identificar los cambios que se produjeron en la organización espacial de esta migración internacional. Insistimos en particular sobre el papel actual del espacio fronterizo, históricamente conocido como lugar de la concentración de la migración de Bolivia a Brasil, y intentamos mostrar de que manera este espacio de frontera se articula tanto con la región metropolitana de São Paulo como con el interior de Bolivia. 


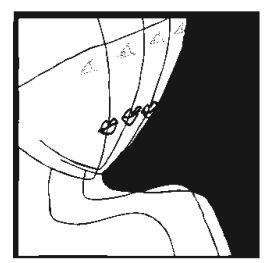

\section{MIGRATIONS SOCIÉTÉ La revue bimestrielle d'analyse et de débat sur les migrations en France et en Europe Janvier-février 2009 vol. $21-n^{\circ} 121-240$ p.}

\section{ÉDITORIAL}

Vers un ministère de l'invasion territoriale, de l'identité nationale... et de la délation "sociale" ? .

Vincent Geisser

\section{ARTICLE}

- Familles des émigrés restées à Oujda

Noureddine Bouammali

DOSSIER : Vers un droit à la mobilité ? (coordonné par Yvain Corradi)

I. INTRODUCTION

- Le droit à la mobilité : nouvel horizon pour penser les migrations?

Yvain Corradi

- Vers un droit universel à la mobilité

Catherine Wihtol de Wenden

II. LES MIGRATIONS AUJOURD'HUI : NOUVELLES FORMES, NOUVELLES CONTRAINTES, NOUVELLES VOIES

- Mascarade des migrations : nouvelles formes, nouveaux enjeux.

Hervé Le Bras

- Mouvements migratoires : différences, diversité et identités.

Michel Wieviorka

- Conséquences des restrictions du droit à la mobilité sur les droits des migrants........

- Privations de droits et initiatives des migrants algériens sans papiers.

Claire Rodier

- La mobilité dans la Corne de l'Afrique : entre urgence humanitaire et contrainte sécuritaire

III. DROIt À LA MOBILITÉ (ET GOUVERNANCE MONDIALE DES MIGRATIONS)

- L'état du droit à la mobilité .

- Droits des travailleurs migrants : état des lieux

- Les restrictions à la libre circulation des travailleurs.

- Les défis à relever dans une économie mondiale en mutation.

- Pour un espace francophone des migrations

- Vers une gouvernance mondiale des migrations : enjeux, réalités et perspectives...

IV. DES ACteURS, DES ACtIONS ET LEURS PROPOSITIONS

- Le rôle des pouvoirs publics locaux : l'expérience de Nantes

Marie-Thérèse Têtu Delage

Hélène Thiollet

Michel Miaille

Jean-François Trogrlic

Dimitrios Kontizas

Jean-Philippe Chauzy

Jean-Pierre Hoss

Mélodie Beaujeu

Jean-Philippe Magnen

Patricia Dupré-Cormerais

- Comment encourager la mobilité des salariés?

Denis Granger

Jean-Louis Malys

- Quel(s) droit(s) pour quelle(s) mobilité(s)?

David Lopez

Conny Reuter

- L'engagement de la société civile

Régine De Clercq

- Gouvernance globale de la migration

- L'apport du Forum mondial sur la migration et le développement.

Romeo E. Matsas

V. SYNTHÈSE

- Le droit à la mobilité. Conclusion du colloque sur le droit à la mobilité. Bibliographie sélective.

Frédéric Tiberghien Christine Pelloquin

\section{NOTE DE LECTURE}

Traces de luttes. 1924-2007. Mon engagement entre utopie et réalité (de Marius Apostolo).

Luca Marin

DOCUMENTATION

Christine Pelloquin

Abonnements - diffusion : CIEMI : 46, rue de Montreuil - 750II Paris

Tél. : 0I 4372 ol 40 ou 0I 43724934 / Fax : 0143720642

E-mail : contact@ciemi.org / Siteweb : www.ciemi.org

France : $44 €$ Étranger : $54 €$ Soutien : $70 €$ Ce numéro : $14 €$ 